\title{
Hemodynamic and Metabolic Observations Associated with Intracoronary Stenting for Acute Closure Following Percutaneous Transluminal Coronary Angioplasty
}

\author{
IVAN K. DE SCHEERDER, M.D., Ph.D., BERNARDINO TUCCILLO, M.D., \\ BRADLEY H. STRAUSS, M.D., PIM J. DE FEYTER, M.D., \\ and PATRICK W. SERRUYS, M.D. \\ From the Thoraxcenter, University Hospital Dijkzigt, Rotterdam, The Netherlands
}

Emergency stent implantation appears to be an effective method for restoring antegrade flow in case of an abrupt coronary occlusion during percutaneous transluminal coronary angioplasty (PTCA). In this case report, hemodynamic and metabolic changes throughout abrupt coronary closure and stent implantation were followed in order to study the efficacy of this bail out technique in restoring metabolic and hemodynamic disturbances due to acute coronary occlusion. (J Interven Cardiol 1991:4:1)

\section{Introduction}

Abrupt coronary occlusion is an unpredictable event which is usually related to a localized dissection of the arterial wall, and occasionally associated with intravascular thrombosis and vasospasm.' When redilatation fails, the recommended management is emergency surgery, but this procedure is associated with a high perioperative mortality and morbidity. ${ }^{2}$ Previous studies report an incidence of myocardial infarction of $31 \%-71 \%$ due to the unavoidable delay between vessel occlusion and surgical revascularization. ${ }^{3}$ Several bail out techniques have recently been proposed to restore the anterograde flow in the setting of abrupt total occlusion, including bail out perfusion catheters, perfusion balloon (Stack, Advanced Cardiovascular Systems, Santa Clara, CA, USA), laser balloon angioplasty, and atherectomy. Emergency stent im-

B. Tuccillo, M.D., is supported by CNR Research Fellowship 203.04.12; and B.H. Strauss, M.D., is supported by the Canadian Heart Foundation.

Address for reprints: P.W. Serruys, M.D., Catheterization Laboratory, Thoraxcenter, Erasmus University, P.0. Box 1738,3000 DR Rotterdam, The Netherlands. Fax: 31-104365192. plantation appears to be an alternative technique capable of restoring anterograde flow and thus stabilizing the clinical condition. ${ }^{4}$ In this article, we describe a patient who required emergency stent implantation as a bridge to coronary bypass surgery following percutaneous transluminal coronary angioplasty (PTCA). Since he had agreed to participate in a PTCA study protocol, we were able to examine hemodynamic and metabolic changes throughout the procedure, including the stent implantation, which provided us with the opportunity to study the efficacy of this bail out technique in restoring metabolic and hemodynamic disturbances due to acute coronary occlusion.

\section{Case Report}

Coronary angioplasty of a proximal left anterior descending lesion (Fig. 1A) was performed in a 45-year-old male with stable angina pectoris. According to our research protocol, we performed four consecutive balloon inflations of 90 seconds with a $3.0 \mathrm{~mm}$ balloon with deflation intervals of 3 minutes. Since the initial result of the procedure was inadequate showing a residual di- 
DE SCHEERDER, ET AL.
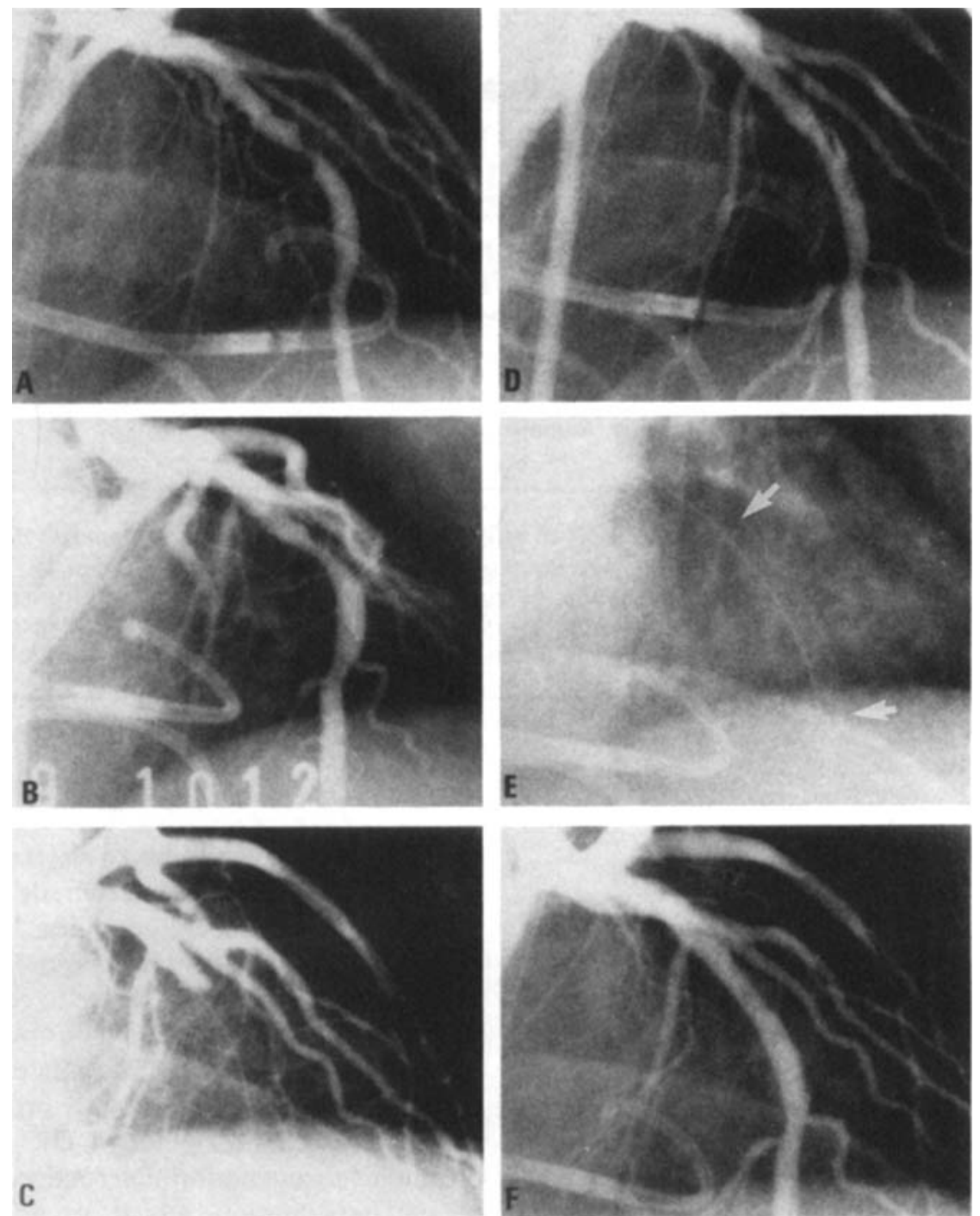

Figure 1. Coronary angiographic view of the left descending artery of our patient before PTCA (A), after inflation with the 3.5 balloon (B), the first total occlusion (C), the dissection $(D)$, the stent $(E)$, and after stent implantation (F).

ameter stenosis $>50 \%$, we repeated the dilatation using a slightly oversized balloon $(3.5 \mathrm{~mm})$, which resulted in a satisfactory outcome (Fig. 1B). However, 6 minutes later the patient experienced acute chest pain, associated with ST elevation in the anterior leads, and abrupt decline of systolic pressure and derived function indices (left ventricular end-diastolic pressure [LVEDP], positive $\mathrm{dP} / \mathrm{dt}$, and $\mathrm{V}_{\max }$ ). Coronary angiography revealed a total occlusion of the dilated vessel (Fig. 1C). Repeat dilatation caused a dissection of the vessel (Fig. 1D). After 20 minutes the vessel reoccluded and a new inflation with a $3.7 \mathrm{~mm}$ balloon was performed, but after 15 minutes total occlusion recurred. After consultation with our standby cardiovascular team, we decided to implant a self-expanding Wallstent ${ }^{\mathrm{R}}$ (Medinvent, $^{\text {(M) }}$ Lausanne, Switzerland) in the coronary artery (Fig. 1E). The stent implantation resulted in an excellent reopening of the vessel (Fig. 1F). A subsequent control angiogram showed incomplete expansion of the endovascular device, and there- 
fore we inserted a $3.0 \mathrm{~mm}$ balloon into the stent and proceeded to inflate within the stent ("Swiss Kiss"). Following completion of left ventriculography and hemodynamic studies, the patient was sent to surgery, according to the institutional protocol for stenting in the setting of acute closure, under stable hemodynamic conditions, with normalized electrocardiogram and without symptoms of angina pectoris. The left internal mammary artery was grafted to the left anterior descending coronary artery and the stent was surgically removed through a left anterior descending arteriotomy. After surgery, no indices (new $Q$ wave, significant enzymes elevation) suggesting a perioperative myocardial infarction were observed.

\section{Hemodynamics}

During the PTCA procedure, left ventricular pressure was measured by a Millar catheter (Millar Instrument, Inc., Houston, TX, USA) and digitized at 250 samples per second. Peak left ventricular pressure, left ventricular diastolic pressure, peak negative $\mathrm{dP} / \mathrm{dt}$, peak positive $\mathrm{dP} /$ $\mathrm{dt}$, and relation between $\mathrm{dP} / \mathrm{dt}$ pressure and pressure linearly extrapolated to pressure $0\left(\mathrm{~V}_{\max }\right)$ were computed for each recorded beat. $\tau_{1}$, the time constant of relaxation period for the first $\mathbf{4 0}$ msec, was also recorded. ${ }^{5}$ This beat-to-beat analysis was performed at the following times: baseline; before, during, and after each balloon inflation; during and after spontaneous coronary occlusions; before, during, and after stent implantation; and before sending the patient to surgery. Leads I, II, III, and $V_{3}$ were continuously monitored during the procedure. Ventriculography was performed before and after PTC $A$ and stent implantation. After the end-diastolic and end-systolic frames were determined, stroke volume, global ejection fraction, and total cardiac index were computed. The regional left ventricular function, and the contribution of each particular segment to global ejection fraction was measured. ${ }^{6}$

Table 1 shows the evolution of left ventricular peak systolic pressure, left ventricular end-diastolic pressure, $\mathrm{dP} / \mathrm{dt}, \mathrm{V}_{\max }$, and $\tau_{1}$ during the procedure. The abrupt changes of all the param-

Table 1.

\begin{tabular}{|c|c|c|c|c|c|c|c|}
\hline & $\begin{array}{c}\text { Time } \\
\text { (minutes) }\end{array}$ & $\begin{array}{c}\mathrm{HR} \\
\text { (beats/min) }\end{array}$ & $\begin{array}{l}\text { LVEDP } \\
(\mathrm{mmHg})\end{array}$ & $\underset{(\mathrm{mmHg})}{\mathrm{SBP}}$ & $\begin{array}{c}+\mathrm{dP} / \mathrm{dt} \\
\text { (mmHg/seconds) }\end{array}$ & $\begin{array}{l}V_{\max } \\
\left(s^{-1}\right)\end{array}$ & $\begin{array}{c}\tau_{1} \\
(\mathrm{msec})\end{array}$ \\
\hline Baseline I & 0 & 82 & 9 & 118 & 1690 & 55 & 40 \\
\hline Baseline II & 10 & 80 & 8 & 115 & 1770 & 61 & 41 \\
\hline Inflation I & 25 & 82 & 13 & 106 & 1330 & 48 & 61 \\
\hline Postinflation I & 27 & 82 & 7 & 117 & 2080 & 73 & 46 \\
\hline Inflation II & 29 & 78 & 12 & 101 & 1250 & 43 & 63 \\
\hline Postinflation II & 31 & 80 & 8 & 118 & 2050 & 71 & 49 \\
\hline Inflation III & 33 & 82 & 13 & 99 & 1180 & 39 & 66 \\
\hline Postinflation III & 35 & 82 & 5 & 114 & 1980 & 72 & 43 \\
\hline Inflation IV & 37. & 85 & 11 & 101 & 1120 & 34 & 65 \\
\hline Postinflation IV & 39 & 85 & 7 & 116 & 1950 & 68 & 47 \\
\hline Postinflation V & 49 & 83 & 7 & 115 & 2010 & 71 & 52 \\
\hline Before spontaneous occlusion & 51 & 71 & 8 & 117 & 1990 & 69 & 51 \\
\hline Spontaneous occlusion I ( 7 minutes) & 60 & 80 & 14 & 86 & 1240 & 52 & 60 \\
\hline Reopening & 71 & 83 & 16 & 106 & 1920 & 74 & 60 \\
\hline Before spontaneous occlusion II & 87 & 88 & 10 & 106 & 1620 & 59 & 50 \\
\hline Spontaneous occlusion II & 90 & 90 & 17 & 105 & 1460 & 51 & 73 \\
\hline Reopening & 101 & 100 & 8 & 116 & 1850 & 64 & 47 \\
\hline Poststent & 141 & 97 & 7 & 116 & 1950 & 68 & 49 \\
\hline Pre-CABG & 176 & 100 & 9 & 113 & 1960 & 70 & 42 \\
\hline
\end{tabular}

Evolution of heart rate (HR), left ventricular end-diastolic pressure (LVEDP), left ventricular peak systolic pressure (SBP), peak positive $\mathrm{dP} / \mathrm{dt}(+\mathrm{dP} / \mathrm{dt})$, relation between $\mathrm{dP} / \mathrm{dt}$ and pressure linearly extrapolated to pressure $0\left(\mathrm{~V}_{\max }\right)$, and the time constant of relaxation period for the first $40 \mathrm{msec}\left(\tau_{1}\right)$ during the study. 
eters during the total occlusion of the coronary artery clearly shows the impairment of left ventricular function. However, after stent implantation, all indices return to baseline values, confirming complete functional recovery of the myocardium. Global left ventricular ejection fraction and the contribution of the anterobasal and anterolateral wall were, respectively, $65 \%, 19.7 \%$, and $9.5 \%$ at baseline, and $71.4 \%, 22 \%$, and $10.5 \%$ following stent implantation immediately prior to surgery.

\section{Metabolism}

To evaluate ATP breakdown during ballooninduced and spontaneous coronary occlusions, we determined hypoxanthine V-A difference. ${ }^{6} \mathrm{~A}$ Pepine catheter (Wilton Webster, Altadena, CA, USA) was inserted in the great cardiac vein for subselective regional venous blood sampling. Hypoxanthine $\mathrm{V}-\mathrm{A}$ difference increased during PTCA, particularly prior to the completion of the fifth balloon inflation (Fig. 2). Hypoxanthine production peaked to a maximum of $9.432 \mu \mathrm{mol}$ at the termination of the second spontaneous coronary occlusion. Before sending the patient to surgery, hypoxanthine $\mathrm{V}$-A difference $(0.221$ $\mu \mathrm{mol}$ ) had returned to baseline values. Biochemical markers of cardiac necrosis (serum creatine, creatine kinase, and creatine kinase MB isoenzyme) were determined before, during, after PTCA and stent implantation, and remained normal throughout the entire procedure.

\section{Discussion}

The goals of therapy for abrupt coronary occlusion after PTCA, namely restoring antero-

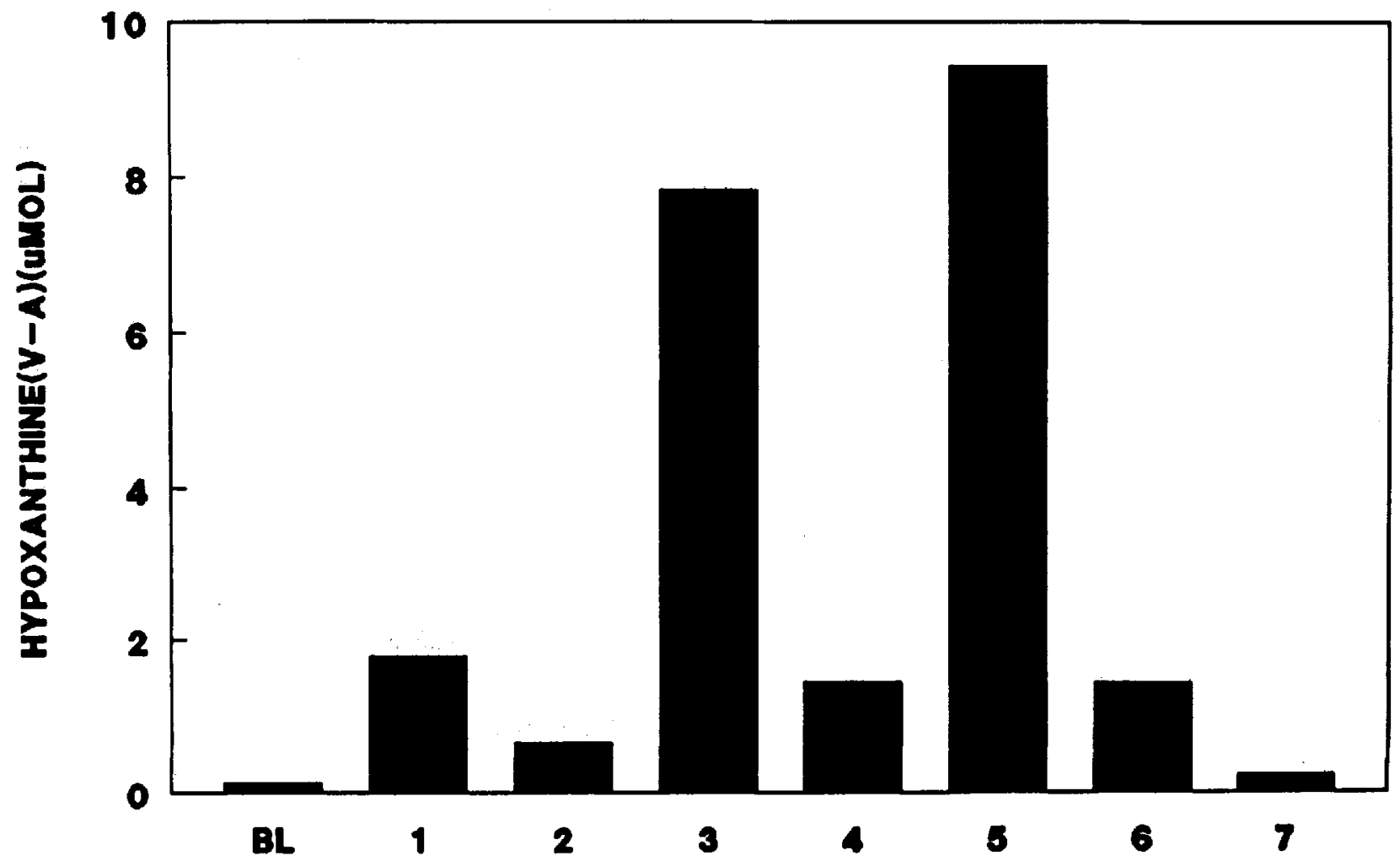

Figure 2. Hypoxanthine V-A difference during baseline (BL), during the fifth PTCA inflation (1), before the first spontaneous occlusion (2), at the end of the first spontaneous occlusion (3), before the second spontaneous occlusion (4), at the end of the second spontaneous occlusion (5), during stent implantation (6), and before operation (7). 


\section{STENTING FOR ACUTE CLOSURE AFTER BALLOON ANGIOPLASTY}

grade flow and preventing recurrences, have conventionally been attempted by repeat dilatations occasionally with oversized balloons or prolonged balloon inflations. If thrombosis is suspected, lytic therapy with thrombolytic agents in addition to redilatation, may be effective in selected patients. If patency of the vessel can not be maintained, other alternatives such as perfusion catheters, Stack perfusion balloon, atherectomy, and laser balloon angioplasty may be considered, otherwise urgent surgical intervention is required. Rapid restoration of adequate myocardial blood flow is best achieved in these emergency surgical cases with saphenous vein grafts, due to their relatively uncomplicated preparation. Although internal mammary arteries are preferred as bypass conduits, the surgical preparation may be too time consuming for emergency situations, particularly in unstable patients. In fact, previous studies report that preoperative ischemia was a significant feature of postprocedural morbidity. The incidence of periprocedural $Q$ wave infarction was only $4 \%$ in patients without preoperative ischemia, but rose to $27 \%$ in emergency coronary artery bypass surgery. In-hospital mortality in patients with preoperative ischemia varies between $0 \%-15 \%{ }^{3}$ Emergency coronary stenting is technically feasible, and can effectively restore anterograde blood flow. It is associated with an acceptable incidence of complications, and may prevent myocardial infarction. This was demonstrated in this report by reversal of biochemical markers of ischemia (hypoxanthine production) without evidence of irreversible myocardial necrosis despite multiple prolonged spontaneous occlusions lasting up to 13 minutes.

The analysis of left ventricular function during the procedure showed marked impairment of systolic and diastolic function during the spontaneous occlusions of the vessel. However, after restoring the anterograde blood flow with stent implantation, total recovery of myocardial function was demonstrated. This occurred despite significant release of purines, indicating that acutely decreasing myocardial ATP content does not necessarily affect cardiac function.
Furthermore, our report confirms the potential value of emergency stent implantation as a bridge to coronary bypass surgery. ${ }^{4}$ Stable hemodynamic conditions enable the surgeon to choose the optimal surgical strategy (e.g., use of an internal thoracic artery as in our patient) and to work safely on a nonemergency basis, decreasing the operative morbidity. Obviously, the placement of a stent is only indicated in selected patients, particularly when abrupt coronary occlusion is associated with a major dissection, intimal hemorrhage, or elastic recoil either occurring alone or in combination. Caution is advisable if intraluminal thrombosis is suspected to be the predominant mechanism, and should be considered as a relative contraindication to stenting.

\section{Conclusion}

The emergency intracoronary stent implantation may be an effective "bail out" device for treatment of refractory coronary occlusion after failed PTCA. It reduces myocardial ischemia, restores hemodynamic stability, and may potentially reduce morbidity and mortality related to emergency coronary artery bypass surgery.

\section{References}

1. Ellis SG, Roubin GS, King III SB, et al. In hospital cardiac mortality after acute closure after coronary angioplasty: Analysis of risk factors from 8,207 procedures. J Am Coll Cardiol 1988; 11:211-216.

2. Page US, Okies JE, Colburn LQ, et al. Percutaneous transluminal coronary angioplasty: A growing surgical problem. J Thorac Cardiovasc Surgery 1986; 92:847-852.

3. Reul GJ, Cooley DA, Hallman GL, et al. Coronary artery bypass for unsuccessful percutaneous transluminal coronary angioplasty. J Thorac Cardiovasc Surg 1984; 88:685-694.

4. Sigwart U, Urban P, Golf S, et al. Emergency stenting for acute occlusion after coronary balloon angioplasty. Circulation 1988; 78:1121-1127.

5. Serruys PW, Wijns W, Van den Brand $M$, et al. Left ventricular performance regional blood flow, wall motion, and lactate metabolism during transluminal angioplasty. Circulation 1984; 70:25-36.

6. Serruys PW, Suryapranata H, Piscione F, et al. Myocardial release of hypoxanthine and lactate during percutaneous transluminal coronary angioplasty. Am J Cardiol 1989; 63:45E-51E. 